\title{
Autologous Mesenchymal Stem Cells-Poly Lactic-co-glycolic Acid
}

National Cancer Institute

\section{Source}

National Cancer Institute. Autologous Mesenchymal Stem Cells-Poly Lactic-co-glycolic

Acid. NCI Thesaurus. Code C148189.

A preparation of autologous bone marrow derived mesenchymal stem cells (MSC) that are seeded on biodegradable poly lactic-co-glycolic acid (PLGA)-containing polymer scaffolds, that can potentially be used for bone marrow engraftment. When administered into the bone lesion of the patient, the autologous MSC-PLGA can be used for bone marrow eng raftment. 